\title{
INFLUENCE OF ELECTRODE GEOMETRY AND HLLS FIT ANALYSIS OF I-V MEASUREMENTS IN A THREE-ELECTRODE CELL
}

\author{
B A. BOUKAMP, I.C. VINKE, K. SESHAN, K.J. DE VRIES and A.J. BURGGRAAF \\ Department of Inorgcnic Chemistry, Materials Science and Catalysis, University of 7 ivente, \\ P.O. Box 217, 7500 AE Enschede, The Netherlcizds
}

Received 10 August 1987

\begin{abstract}
The analysis of electrorle polarisation ( $I-V$ ) measurernents of oxygen electrodes on $\varepsilon \cdot \mathrm{Bi}_{2} \mathrm{O}_{3}$-oased solid electrolytes is complicated by an ohmic polarisatın correction which is of the order of the electrode resistance. The analysis can be performed with a NLLS fit technique, which inciudes this correction resistance, $R_{\mathrm{u}}$, as adjustable parameter. By an appropriate choice of the electrode geometry the factor $R_{\mathrm{u}}$ can be minimized.
\end{abstract}

\section{Introduction}

The application of oxygen ion sonducting solid electrolytes in electrochemical devices, such as fuel cells, oxygen pumps and electro-cataiytic reactors, depends on the availability of good, stable oxygen electrodes that exhibit high exchange currents. Recent results on $\delta-\mathrm{Bi}_{2} \mathrm{O}_{3}$-based solid electrolytes have show: that these materials have a high surface oxygen exchange current, independent of the type of (noble) metal electrode applied [1].

The properties of these porous gold and platinum electrodes and of thin mixed conducting oxide electrodes on such electrolytes are measured in a threeelectrode cell [2-5] allowing the anodic and cathodic polarisation to be measured separately. In most cases the current-overvoltage relation can be expressed in terms of the Butler-Volmer equaticn (BV),

$I=I_{0}\left[\exp \left(\alpha_{\mathrm{a}} n F \eta / R T\right)-\exp \left(-\alpha_{\mathrm{c}} n F \eta / R T\right)\right]$,

where $n$ is the number of times the rate detemining step has to occur for the complete reaction to take

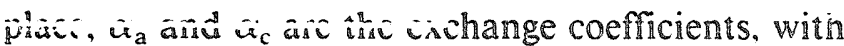
$\alpha_{a}+\alpha_{c}=1$, and $\eta$ is the actual overpotential of the electrode, which is measured with respect to a (currentless) reference electrode. Because of the current flux lines between the work and counter electrode and the position of the reference electrode an ohmic potential drop exists between the reference anc' work electrode for which $\eta$ must be corrected.

$\eta=V-R_{\mathrm{u}}$

$V$ is the actual voltage measured between work and reference electrodes. $\boldsymbol{R}_{\mathrm{u}}$ depends in principle on the bulk resistivity of the electrolyte and the geometric arrar gement of the electrodes. In many cases $R_{\mathrm{u}}$ can be neglicied with little error as it is small compared to the electrode resistance, $R_{\mathrm{e}}$,

$R_{\mathrm{e}}=\mathrm{d} V /\left.\mathrm{d} I\right|_{V=0}=R T / n F I_{0}$.

For the currently investigated Bi-containing electrolytes $[1,6]$ the exchange currents are so high that $R_{\mathrm{e}}$ becomes of the same order of $\boldsymbol{R}_{\mathrm{u}}$ in magnitude and thus the correction criticaliy influences the calculated parameters for the BV equation. To improve this situation cne can optimise the electrode geometry and obtain the $R_{\mathrm{u}}$ from ac impedance measurements in the three-electrode cell.

Due î́ ine limited voltage range thrô can be applied to these materials, the anodic branch of the polirisation often does not reach, after correction for $\mathbb{R}_{\mathrm{u}}$, into the linear region in the Tafel plot $(\ln |\tilde{n}|$ versus $\eta$ ). This can be overcome by analysing the $I-V$ data with a NLLS fit procedure which includes $R_{\mathrm{u}}$ as adjustable parameter. The potential of the NLLS fitting techniques has recently been demonstrated in

0 167-2738/88/\$03.50 (C) Elsevier Science Publishers B.V.

(North-Holland Physics Publishing Division) 
impedance spectroscopy and is recognised as a powerful tool in data analysis $[7,8]$.

\section{Optimizing the electrode geometry}

On thin, disc-shaped samples the work electrode and the (noble metal) reference electrode are situated on one side of the sample. For practical reasons a well-deîned, annular-shaped work electrode is used with the reference point electrode in its center, while the opposite face of the solid electrolyte disc is almost covered with a full circular counter electrode. A two-dimensional simulation for this arrangement, using conductive paper, shows that the potential of the reference electrode approaches the counter electrode potential (fig. 1A). Using also an annularshaped counter electrode increases the reference electrode potential above half the difference between the counter and work electrode (fig. IB), and consequently decreases $R_{\mathrm{u}}$. The value of $R_{\mathrm{u}}$ depends strongly on the atio of the inner radius, $r_{\mathrm{a}}$ of the annular electrodi and the thickness of the sample. Therefore it is advisable to decrease $r_{\mathrm{a}}$ as much as is practically feasible.
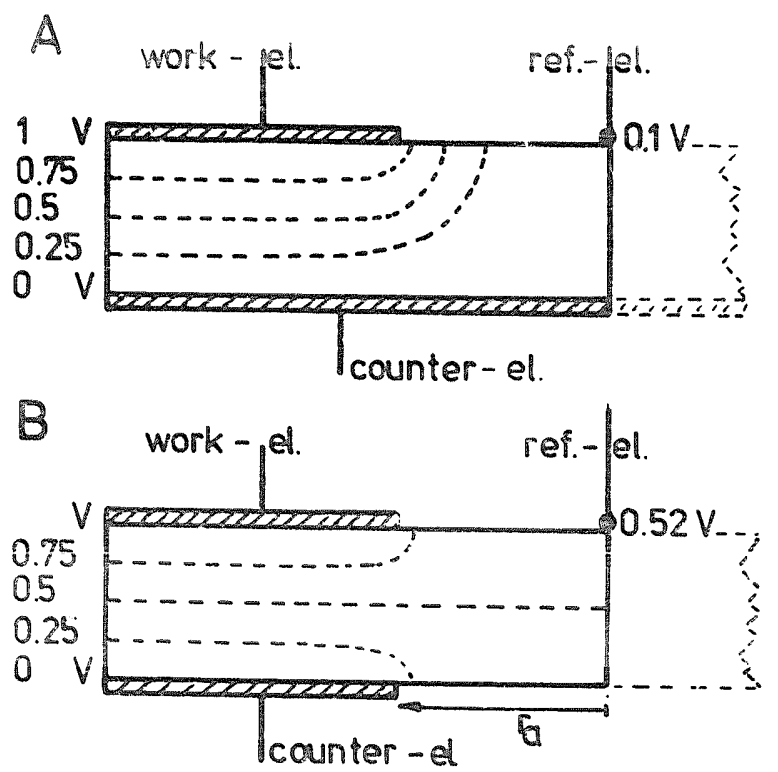

Fig. 1. Schematic two-dimensional model for the equipotential lines for (A) a full counter electrode, and (B) an annular counter clectrode. Resulis are obtained with conductive paper.

\section{NLLS fit procedure}

Fitiing a model function $F\left(x, a_{j}\right), j=1, \ldots, m$, to a set of measured data, $x_{i}, y_{i}, i=1, \ldots, N$, is a well-known procedure. A large variety of NLLS fitting routines are available in program libraries, both for main frame and personal computers. In such a procedure, the weighted error sum

$S=\sum_{i=1}^{N} w_{i}\left[y_{i}-F\left(x_{i}, a_{j}\right)\right]^{2}$

is minimised by simultaneously adjusting the parameters $a_{j}$ of the fitting function. As $F\left(x, a_{j}\right)$ is generally not linear in its parameters, it is linearised through a Taylor series development around a set of starting values, $a_{j}^{0}$, ignoring higher-order terms. By setting the partial derivatives of the error sum with respect to the parameters, $\partial S / \partial a_{j}$, to zero, a set of $m$ linear equations is obtained. In matrix form:

$\boldsymbol{\alpha} \cdot \mathbf{i} \boldsymbol{\alpha}=\boldsymbol{\beta}$

with

$\alpha_{m: k}=\sum_{i=1}^{N} w_{i} \frac{\partial F\left(x_{i}, a_{j}\right)}{\partial a_{m}} \frac{\partial F\left(x_{i}, a_{j}\right)}{\partial a_{k}}$

and

$\beta_{h}=\sum_{i=1}^{\Lambda} w_{l}\left[y_{l}-F\left(x_{l}, a_{j}\right)\right] \frac{\partial F\left(x_{i}, a_{j}\right)}{\partial a_{k}}$.

By multiplying botn sides with the inverse of matrix $a, \epsilon=\mathbb{a}^{-1}$, the solution is found. This results in a new, improved set of (starting) parameters. This iterative procedure is continued until the change in the parameters becomes insignificant [7,9].

For the $\mathbb{I}-V$ measurements the fit function becomes the modified Butler-Volmer equation, with $A=n F / R T$,

$$
\begin{aligned}
I= & I_{0}\left\{\exp \left[\alpha_{\mathrm{a}} A\left(V-R_{\mathrm{u}}\right)\right]\right. \\
& \left.\left.-\exp \left[-\left(1-\alpha_{\mathrm{a}}\right) A\left(V-\mathbb{I} \mathbb{R}_{\mathrm{u}}\right]\right)\right]\right\},
\end{aligned}
$$

which imposes a probiem as it cannot be expressed simply in terms of a $f_{1} t$ function, $F\left(V, \alpha_{a}, I_{0}, R_{u}\right)$, because $I$ appears in both exponents on the right-hand side of eq. (8). The evaluation of $I$ for a set of $\alpha_{a}, I_{0}, R_{u}$ at a specified voltage, $V$, can be obtained, however, through an iterative procedure. For this a new function, $f(I)$, is defined: 


$$
\begin{aligned}
& f(I)=I-I_{0}\left\{\exp \left[\alpha_{\mathrm{a}} A\left(V-I R_{\mathrm{u}}\right)\right]\right. \\
&\left.-\exp \left[-\left(1-\alpha_{\mathrm{a}}\right) A\left(V-I R_{\mathrm{u}}\right)\right]\right\} .
\end{aligned}
$$

For $f(I)=0$ the correct value for $I$ is found. The root of $f(I)$ can be obtained using a Newton-Raphson iteration. For a starting point, $I^{\prime}$ on $f(I)$ the tangential line is drawn. The intersection of this line with the $I$-axis gives a new improved value, $I^{\prime \prime}$,

$I^{\prime \prime}=I^{\prime}-f\left(I^{\prime}\right)\left[\mathrm{d} f(I) /\left.\mathrm{d} I\right|_{l=r^{\prime}}\right]$.

This iteration is carried out until the change in $I$ becomes insignificant.

The NLLS fit proced:ise also requires the partial derivatives with respeci to the parameters $\alpha_{\mathrm{a}}, I_{0}, R_{\mathrm{u}}$ of $I(V)$, (eqs. $(6,7))$. These can be obtained directly from the modified BV, eq. (8). E.g. for $\partial I / \partial I_{0}$,

$$
\begin{aligned}
& \partial I / \partial I_{0}=\left(e^{+}-e^{-}\right)-I_{0}\left[e^{+} \alpha_{\mathrm{a}} A R_{\mathrm{u}} \partial I / \partial I_{0}\right. \\
& \left.\quad+e^{-}\left(1-\alpha_{\mathrm{a}}\right) A R_{\mathrm{u}} \partial I / \partial I_{0}\right]
\end{aligned}
$$

with

$e^{+}=\exp \left[\alpha_{\mathrm{a}} A\left(V-I R_{\mathrm{u}}\right)\right]$,

$e^{-}=\exp \left[-\left(1-\alpha_{\mathrm{a}}\right) A\left(V-\mathbb{R}_{\mathrm{u}}\right)\right]$.

Bringing $\partial I / \partial I_{0}$ to the left-hand side results in

$\partial I / \partial I_{0}=\left(e^{+}-e^{-}\right) / Q$,

with

$Q=1+I_{0} A R_{\mathrm{u}}\left[\alpha_{\mathrm{a}} e^{+}+\left(1-\alpha_{\mathrm{a}}\right) e^{-}\right]$.

In the same way the other twe oartial derivatives are found:

$\partial I / \partial \alpha_{\mathrm{a}}=I_{0} A\left(V-\mathbb{R} \mathbb{R}_{\mathrm{u}}\right)\left(e^{+}-e^{-}\right) / Q$

and

$\partial I / \partial R_{\mathrm{u}}=-I_{0} I A\left[\alpha_{\mathrm{a}} e^{+}+\left(1-\alpha_{\mathrm{a}}\right) e^{-}\right] / Q$.

With this set of formulas the NLLS fit can easily be programmed on a personal computer.

The fit procedure requires a set of staring values for the adjustable parameters. This can readily be automated as only one function is used in the analysis. For most of the oxygen electrodes on solid electrolytes the anodic polarisation is much less than the cathodic one. As a consequence, the influence of $R_{0}$ is noticed mostly in the anodic branch. Hence the slope at the high voltage limit of the anodic branch of the $I-V /$ curve gives an estimate for $R_{\mathrm{u}}$, while the slope of the curve at $V=0$ gives the sum of $R_{\mathrm{e}}$ and $\mathbb{R}_{\mathrm{u}}$ :

$\mathrm{d} I /\left.\mathrm{d} V\right|_{V \rightarrow \infty}=1 / R_{\mathrm{u}}$,

$\mathrm{d} I /\left.\mathrm{d} V\right|_{V=0}=1 /\left(R_{\mathrm{u}}+R_{\mathrm{c}}\right)$.

Using eq. (3) an estimate for $I_{0}$ is obtained from $R_{\mathrm{e}}$, while the temperature and $n$ are specified by the user. The estimate for $\alpha_{\mathrm{a}}$ is obtained from the Tafel slope of the cathodic branch, after correction for $R_{\mathrm{u}}$. The quality of the starting values obtained thus generally allows a simple analytical NLLS fit, which converges faster than the Marquardt NLLS fit procedure $[8,10]$. An added advantage of this fit procedure is that under certain assumptions error estimates may be obtained for tile parameters from the error matrix, $\epsilon$ [7].

\section{Four-parameter fit procedure}

In quite a number of $I-V$ measurements, performed with porous gold electrodes on $\left(\mathrm{Bi}_{0.75}\right.$, $\left.\mathrm{Er}_{0.25}\right)_{2} \mathrm{O}_{3}$ electrolyte (BE25), it was obvicus that integer values for $n$ did not esult in adequate fits [6]. Because of this the fit procedure was augmented to include also the parameter $n$. For practical reasons the anodic and sathodic exchange coefficients were then defined as

$\alpha_{\mathrm{a}}^{\prime}=\alpha_{\mathrm{a}} n$ and $\alpha_{\mathrm{c}}^{\prime}=\alpha_{\mathrm{c}} n=\left(1-\alpha_{\mathrm{a}}\right) n$.

Also the derivatives must be modified and extended:

$\partial / \partial \alpha_{\mathrm{a}}=I_{0} A\left(V-I R_{\mathrm{u}}\right) e^{+} / Q$,

$\partial I / \partial \alpha_{\mathrm{c}}=I_{0} A\left(V-\mathbb{R}_{\mathrm{u}}\right) e^{-1 Q}$,

$\partial I / \partial I_{0}=\left(e^{+}-e^{-}\right) / Q$

and

$\exists I / \partial \mathbb{R}_{\mathrm{u}}=-\mathbb{I}_{0} \mathbb{A}\left(\alpha_{\mathrm{a}} e^{+}+\alpha_{\mathrm{c}} e^{-}\right) / Q$,

with

$Q=1+\mathbb{I}_{0} A R_{\mathrm{u}}\left(\alpha_{\mathrm{a}} e^{+}+\alpha_{\mathrm{c}} e^{-}\right)$.

The results of the three-parameter fir present excellent starting values for a subsequent four-parameter fit procedure, which then only requires an analytical nit procedure. 


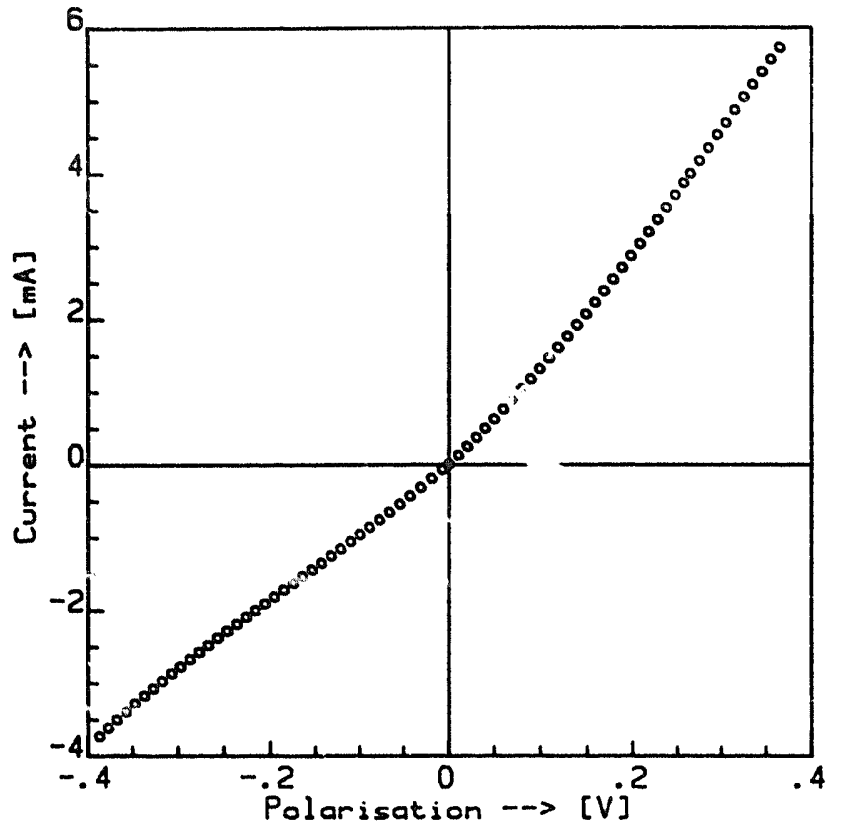

Fig. 2. $i-V$ curve for a porous gold electrode on $\left(\mathrm{Bi}_{0.75}, \mathrm{Er}_{6.25}\right)_{2} \mathrm{O}_{3}$ in oxygen at $917 \mathrm{~K}$. This curve presents a "worst case curve" where $R_{\mathrm{c}} \approx R_{\mathrm{u}}$.

\section{Results and discussion}

Fig. 2 shows a good example of $I-V$ data where $R_{\mathrm{e}}$ is of the same magnitude as $R_{\mathrm{u}}$. The data are taken from a $\left(\mathrm{Bi}_{0.75}, \mathrm{Er}_{0.25}\right)_{2} \mathrm{O}_{3}$ sample with porous gold

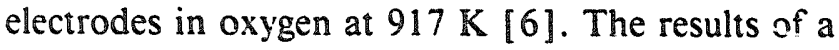
three parameter fit for $n=2$ and $n=3$, and for a fourparameter fit with error estimates are given in table 1. The value, $n=2.6$, obtained from the four-parameter fit is tentatively interpreted as a competition between a charge-transfer-limited process $(n=2)$ and

Table 1

Results for a three- and four-parameter NLLS fit procedure for the data of fig. 2. For the three-parameter fit the value of $n$ was specified. Unity weight factors were used in all fit procedures.

\begin{tabular}{lccl}
\hline Parameter & \multicolumn{2}{l}{$\begin{array}{l}\text { Three-parameter } \\
\text { NLLS fit }\end{array}$} & $\begin{array}{l}\text { Four-parameter } \\
\text { NLLS fit }\end{array}$ \\
\cline { 2 - 3 } & $n=2$ & $n=3$ & $\begin{array}{l}\text { ivith estimated } \\
\text { error }\end{array}$ \\
\hline$\alpha_{c}$ & 0.75 & 0.79 & $0.77 \pm 0.03$ \\
$\alpha_{\mathrm{c}}$ & 0.25 & 0.21 & $0.23 \pm 0.01$ \\
$n$ & $(2)$ & $(3)$ & $2.57+0.18$ \\
$\mathbb{f}_{0}(\mathrm{~A})$ & 9.7 & 7.9 & $(8.5 \pm 0.2) \times 10^{-4}$ \\
$\mathbb{R}_{\mathrm{u}}(\mathrm{ohm})$ & 46.4 & 51.9 & $50.0 \pm 0.5$ \\
$\mathbb{R}_{\mathrm{c}}(\mathrm{ohm})$ & 40.7 & 33.4 & $36.2 \pm 1.4$ \\
\hline
\end{tabular}

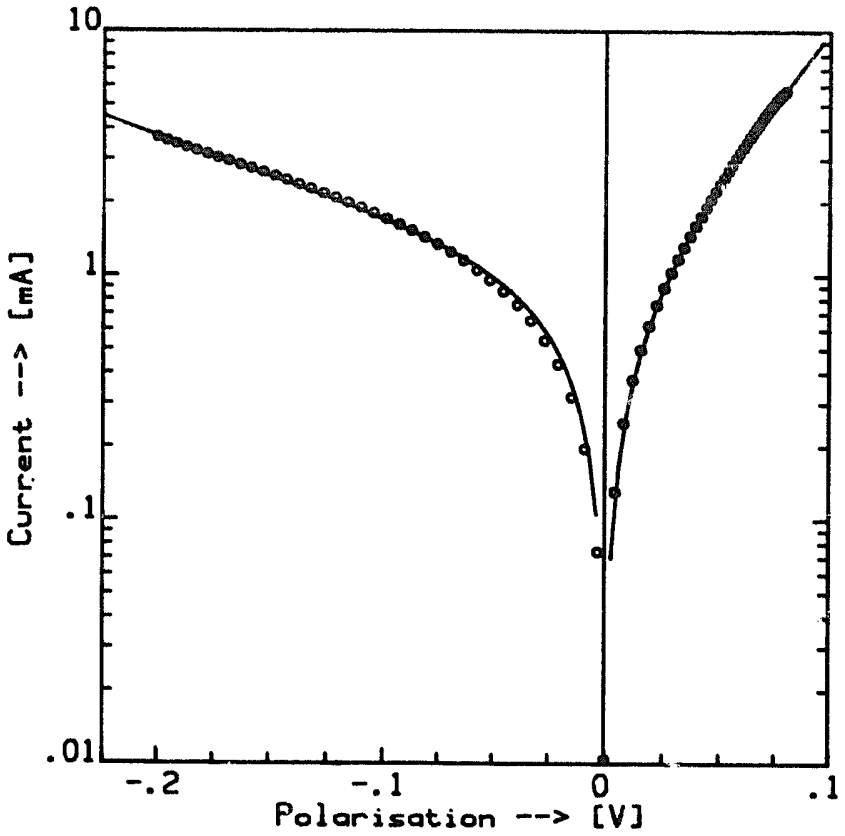

Fig. 3. Tafel plot, corrected for $I R_{\mathrm{u}}$, of the $I-V$ data of fig. 2. The solid line represents the result of the four-parameter NLLS fit.

a diffusion-limited process [ 6 ]. Apparent non-integer values for $n$ have also been found for $\mathrm{Pt}$ electrodes on $\mathrm{Y}_{2} \mathrm{O}_{3}$ stabilised $\mathrm{ZrO}_{2}$ [11].

The correctness of the fit is best observed in the (for $I R_{\mathrm{u}}$ corrected) Tafel plot of fig. 3 . It is obvious that the choice of $R_{\mathrm{u}}$ will strongly influence the observed value for $n$, obtained from a simple graphical anaiysis in a corrected Tafel plot. The weight factors were set to 1 , assuming that the statistical errors in the measured data are independent of the current or voltage. The reproducibility of the fit results for identical measurements was found to be excellent. The spread in the calculated parameters was close to the calculated error estimates form the NLLS fits.

\section{References}

[1] I.C. Vinke, K. Seshan, B.A. Boukamp, K.I. de Vries and A.J. Burggraaf, Solid State lonics, to be published.

[2] K.J. de Vries, M.P. van Dijk, H. Kruidhof and A.J. Burggraaf, in: Proc. 6th Intern. Symp. Metallurgy and Materials Science, eds. F.W. Poulson et al. (Risø Nat. Lab, Roskilde, 1985) p. 323.

[3] A.J. Burggraaf, M.F. van Dijk and K.J. de Vries, Solid State Ionics $18 / 19$ (1986) 897 .

[4] M.P. van Dijk, K.J. de Vries and A.J. Burggraaf, Solid State lonics $21(1986) 73$. 
[5] M.P. van Dijk, K.J. de Vries and A.J. Burggraaf, Solid State Ionics 21 (1986) 83.

[6] I.C. Vinke, K. Seshan, K.J. de vries, B.A. Boukamp and A.J. Burggraaf, to be published.

[7] B.A. Boukamp, Solid State Ionics 20 (1986) 31.

[8] J.R. Macdonald, J. Schoonman and A.P. Lehnen, J. Electroanal. Chem. 131 (1982) 77.
[9] P.R. Bevington, Data reduction and error analysis tor the physical sciences (McGraw-Hill, New York, 1969).

[10] D.W. Marquardi, j. Soc. Indian Appl. Math. 11 (1963) 431.

[11] B.C. Ngyen, L.M. Rincon-Rubio and D M. Mason, J. Electrochem. Soc. 133 (1986) 1860. 